\title{
Laparoscopic reduction and repair of a large incarcerated paraesophageal hernia
}

\author{
Salomone Di Saverio MD, Nazareno Smerieri MD
}

$\mathrm{A}$ 67-year-old woman presented to the emergency department with severe dysphagia, persistent vomiting, epigastric pain and abdominal distension. The results of a electrocardiograph were normal, and a nasogastric tube was inserted. Radiographic findings were consistent with incarcerated paraesophageal hiatus hernia with gastric distension (Appendix 1, available www.cmaj.ca/lookup/suppl/doi:10.1503 /cmaj.131333/-/DC1). Computed tomography with water-soluble contrast showed the "upsidedown" stomach radiologic sign (Figure 1), confirming the diagnosis. An incarcerated gastric hernia was reduced into the abdomen during emergency laparoscopy. Given the large size of the hiatal defect, laparoscopic repair was performed with $\mathrm{u}$-shaped prosthetic mesh placement and intracorporeal stitches. The patient's postoperative course was uneventful, and she was discharged six days later.

The incidence of hiatal hernia in the general population is about $5 / 1000$, but almost all $(95 \%)$ of these are small, sliding or type I hernias that are rarely associated with serious complications. ${ }^{1}$ Giant paraesophageal hernia (type II) are uncommon. ${ }^{1}$ Risk factors for paraesophageal hernia include older age, obesity, tobacco use, collagen vascular diseases and diabetes. ${ }^{2}$ Incarceration and obstruction of an intrathoracic stomach are potentially devastating complications, especially if diagnosed and treated late. ${ }^{3}$

Current guidelines suggest that emergency presentations of hiatal hernia including gastric obstruction or ischemia should first be decompressed with a nasogastric tube, followed by plain chest radiography and endoscopy; excessive investigations may lead to a delay in treatment and worse outcomes. ${ }^{4}$ In the case of possible perforation, a water-soluble contrast medium is preferred to barium for radiological imaging.

Gastric decompression and resuscitation are important elements of preoperative management of acutely presenting hiatus hernias but only early

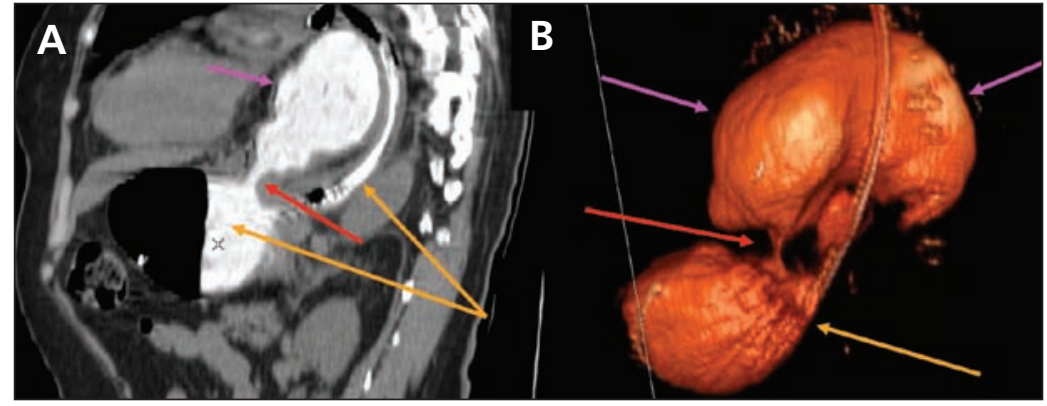

Figure 1: (A) Computed tomography with oral contrast media in a 67-year-old woman showing an "upside-down" stomach sign (organo-axial rotation of the stomach with gastric volvulus resulting in reversed positions of the lesser and greater curvatures). The gastric body, antrum and pylorus are in the chest cavity and rotated, while the cardia and fundus are in the abdomen. (B) 3-Dimensional multiplanar reconstruction showing stricture at the diaphragmatic hiatus level (red arrow). The purple arrows indicate intrathoracic incarcerated stomach and partial gastric volvulus, and the orange arrow indicates the nasogastric tube ending in the gastric fundus, which is located below the diaphragm.

surgery yields better outcomes. Acutely incarcerated paraesophageal hernias have been traditionally managed through emergency open surgery (i.e., through an abdominal or thoracic approach), with accompanying high rates of morbidity and mortality, especially among older people. ${ }^{5}$ Early endoscopy may decompress incarcerated paraesophageal hernias, and emergency laparoscopic reduction is a safe, minimally invasive alternative to traditional surgery.

See references, www.cmaj.ca/lookup/suppl/doi:10.1503/cmaj $.131333 /-/ \mathrm{DC} 1$

Acknowledgements: The authors thank Prof. Elio Jovine, head of the Department of Surgery, and Dr. Barbara Bacer, anesthetist for this case.
Competing interests: None declared.

This article has been peer reviewed.

Affiliation: Maggiore Hospital Regional Emergency Surgery and Trauma Center, Bologna Local Health District Bologna, Italy

Correspondence to: Salomone Di Saverio, salo75@inwind.it

CMAJ 2014. DOI:10.1503 /cmaj.131333
Please see the following video online:

Incarcerated paraesophageal hiatus hernia with "upside-down" stomach: emergency laparoscopy for reduction and mesh repair. www.cmaj.ca/lookup/suppl/doi:10.1503/cmaj.131333/-/DC1. 